\title{
Efficient 3-D electromagnetic modeling procedure of composite materials in microwave frequency range: application to EMC characterization of complex media by statistical means
}

\author{
Sébastien Lalléchère ${ }^{1, *}$ \\ ${ }^{1}$ Université Clermont Auvergne, CNRS, SIGMA Clermont, Institut Pascal, 4 av Blaise Pascal, 63000 Clermont-Fd, France \\ *corresponding author, E-mail: sebastien. lallechere@uca.fr
}

\begin{abstract}
The aim of this proposal is to demonstrate the ability of tridimensional (3-D) electromagnetic modeling tool for the characterization of composite materials in microwave frequency band range. Indeed, an automated procedure is proposed to generate random materials, proceed to 3-D simulations, and compute shielding effectiveness (SE) statistics with finite integration technique (FIT, CST $\AA$ ) microwave studio solver). In this context, 3-D electromagnetic models rely on random locations of conductive inclusions; results are compared with classical electromagnetic mixing theory (EMT) approaches (e.g. Maxwell-Garnett formalism), and dynamic homogenization model (DHM).

The article aims to demonstrate the interest of the proposed approach in various domains such as propagation and electromagnetic compatibility (EMC).
\end{abstract}

\section{Introduction}

\subsection{Physical context}

Nowadays, the need for accurate, efficient, and deterministic electromagnetic tools is all the more important than more and more applications require relevant modeling procedures to simultaneously take into account the complexity (e.g. models needing multi-physics solvers, and multiscales materials) of various complex media. In this framework, the community of metamaterials has deeply questioned the necessity for advanced procedures: for instance regarding the impact of surface roughness of materials on metamaterial performances [1] (jointly with finite difference in time domain, FDTD, simulations), or the introduction of coordinate transformation to design original metamaterial devices such as antennas in [2].

Moreover, due to their heavy use in different industrial fields (such as transport: for instance automotive and/or aerospace), composite materials have experienced a constant interest for electromagnetic purposes from early 2000's (e.g. trade-off between mass and electrical properties grounding, shielding, conductivity...). Thus, different domains including wireless propagation [3] and electromagnetic compatibility [4] have shown a growing interest for theoretical, numerical [5], and/or experimental [6] developments in this framework.

\subsection{Statement of the problem}

Homogenization procedures are nowadays actively used for the macroscopic characterization of materials (i.e. at macroscopic scale, for instance regarding shielding effectiveness, SE). Various works were proposed to extract the properties of composite materials mixing gas, liquid, and solid phases with solid matrix, respectively for metrological control [7], geoscience remote sensing [8], and electromagnetic compatibility (EMC) [4]. In this context, different methods are based upon effective medium theory (EMT, e.g. Maxwell-Garnett, MG, formulation) although the limit of validity of formalisms is often a tricky issue [9]. Thus, Koledintseva et al. detailed main features of MG approximation in [10] for a given mixture: electrodynamic isotropy, linearity (independence to the intensity of electromagnetic (EM) field, independence to time variations according to some external action (e.g. thermal, mechanical...), coupling between inclusions is weak, intrinsic size of inclusions is small relatively to the wavelength in the composite matrix (quasi-static approximation), inclusions are arbitrarily oriented ellipsoids, and in case of conducting inclusions, their volumetric rate is lower than the percolation threshold. The aim of this work is to provide useful EM tool to characterize and/or calibrate approximated (analytical) formulations based upon EMT.

This contribution is organized as follows: firstly, section 2 provides theoretical basis and describes the proposed procedure; based upon various numerical examples; then, section 3 both demonstrates the validity and the interest of the automated tridimensional tool by facing results with theoretical expectations from MG [10] and DHM [4] formalisms. Finally, section 4 proposes concluding remarks and details forthcoming works.

\section{Fundamentals and proposed methodology}

This section is devoted to the presentation of theoretical basis jointly with the description of the whole procedure needed to extract SE statistics of composite mixtures.

\subsection{Theoretical foundations}

Firstly, EMTs are mainly used to avoid costly 3-D modeling of composite materials. Nevertheless, classical mixing formulas may reveal inappropriate (e.g. when increasing 
volumetric rate of inclusion, frequency range, and/or conductivity of inclusions), and advanced mixing procedures were proposed: for instance analytical or semi-analytical developments in $[10,11]$, numerically-based dynamic homogenization in $[4,12,13]$ respectively for optical and microwave frequencies. In these references, the authors have taken into account skin effects of inclusions respectively analytically [10] and numerically [4, 13]. A crucial point relies also on the inherent statistical distributions of the shapes of the inclusions, it is developed respectively with semi-analytical [11] and numerical [12, 13] formulations.

Then, a crucial issue relies on the ability to realistically model statistical variability of constitutive parameters (e.g. locations of inclusions). In this framework, we propose an automated procedure in order to: generate random location of inclusions, launch electromagnetic simulations, and extract shielding strength of materials. Figure 1 details numerical setup developed with CSTß [14] MWS and transient solver (Finite Integration Technique, FIT).

Mixing equations stand for efficient and useful approach for characterizing material composites. Many formulations are available, a synthetic work is proposed in [15] to provide common mixing equations to classical MaxwellGarnet (MG), Bruggeman-Rule (BR), and the coherent potential rule respectively. As expressed in [4], the particular case of biphasic composites (phases $u,(u=1,2))$ as the one expected in this work (i.e. phase 1 for composite matrix and volumetric rate $\eta$, and phase 2 for conducting spherical inclusions of volume fraction $\nu, \eta+\nu=1$ ) leads to a Cartesian $3 \times 3$ depolarization tensor $\mathbf{N}$ such as $N_{i j}=0$ for $i \neq j$ $N_{i i} \equiv N_{i}=1 / 3$ in each direction $i(i=x, y, z)$. Thus, the effective permittivity $\epsilon_{m, i}$ in direction $i$ is given by

$$
\epsilon_{m, i}=\frac{\epsilon_{1} \frac{\eta}{\epsilon_{\infty}+N_{i}\left(\epsilon_{1}-\epsilon_{\infty}\right)}+\epsilon_{2} \frac{\nu}{\epsilon_{\infty}+N_{i}\left(\epsilon_{2}-\epsilon_{\infty}\right)}}{\frac{\eta}{\epsilon_{\infty}+N_{i}\left(\epsilon_{1}-\epsilon_{\infty}\right)}+\frac{\nu}{\epsilon_{\infty}+N_{i}\left(\epsilon_{2}-\epsilon_{\infty}\right)}}
$$

where $\epsilon_{m, i}$ stands for the complex effective permittivity ( $\epsilon=\epsilon^{\prime}-j \epsilon^{\prime \prime}=\epsilon^{\prime}-j(\sigma / \omega)$ for harmonic excitation) of material mixture, $\epsilon_{\infty}$ is the complex permittivity of the infinite medium (assuming particular values for $\epsilon_{\infty}$ leads to different mixing methods). In this work, we consider spherical inclusions; this leads to $N_{i}=1 / 3(i=1,2,3)$. Terms $\epsilon_{1}$ and $\epsilon_{2}$ are complex dielectric permittivities respectively for dielectric matrix (lossless, $\sigma_{1}=0 \mathrm{~S} / \mathrm{m}$ ) and conducting inclusions $\left(\sigma_{2}=1000 \mathrm{~S} / \mathrm{m}\right)$. Based upon relation (1), MG formulation is extracted by assuming $\epsilon_{\infty}=\epsilon_{1}$ (which is the case in low frequency band range, and/or with weak concentration of inclusions), and one may define $\epsilon_{m}$ as follows:

$$
\epsilon_{m, i} \equiv \epsilon_{m}=\epsilon_{1} \frac{\epsilon_{2}(1+2 \nu)-\epsilon_{1}(2 \nu-2)}{\epsilon_{1}(2+\nu)+\epsilon_{2}(1-\nu)} .
$$

Parallely to previous formulation, the mechanical model from Mori-Tanaka [16] was extended in electromagnetics by Preault et al. [4] by taking into account the influence of size of inclusions throughout the following relation

$$
\epsilon_{\infty}=\epsilon_{1}+\epsilon_{2} * \alpha,
$$

where $\alpha$ stands for weight coefficient depending on the typical size $d$ of inclusions (for instance the diameter of cylindrical fibers $[4,17]$, and/or the diameter of spherical inclusions here). Relying on previous assumptions, dynamic homogenization method (DHM) was successfully used in [4] by defining $\alpha=(d / \lambda)^{2}$, where $\lambda$ represents the wavelength of the excitation source in effective medium. Even if some additional formulations exist to predict wave transmission in composite materials, the proposed results will next lay emphasis on classical MG and DHM formulations. Indeed, it is possible to use semi-analytical and/or numerical formulations (for fibers and/or ellipsoids) both taking into account random configurations of composite mixtures $[11,12]$ jointly with skin effect $[10,4]$. In the following, for the illustration, we will assume spherical inclusions with conductivity not greater than $\sigma=1,000 \mathrm{~S} / \mathrm{m}$. The choice of this conductivity level is obviously weaker than the one classically found (e.g. for mixtures based on carbon inclusions with conductivity from ten to sixty times greater than $\sigma$ ), but this remains realistic with regards to a huge majority of materials used for EMC applications. Without any lack of generality, relying on works in $[4,10,11,12]$ would be of great interest jointly with the numerical approach detailed in next subsection.

\subsection{Literature overview and modus operandi of auto- mated process}

\subsubsection{State-of-the-art}

Some recent publications have demonstrated the relevance of numerical tools for characterizing properties of reinforced composite materials: for instance, huge work was provided in $[4,12]$ with finite element method (FEM) and COMSOL Multiphysics $($ ) tool [18]. These papers were based upon bidimensional (2-D) case (circular section of infinite cylinders in one direction) and gave relevant information about the SE of composite material made of different shapes of inclusions (spheroids) [12], and proposed accurate modeling of inclusions in high frequency range (i.e. taking into account size of 2-D circular conductive inclusions jointly with skin effect as it was the case in [10]) with EMC expectations in [4]. Based upon previous reference, some interesting works were recently proposed in [17], reproducing 2-D results with 3-D frequency solver (FEKO EM simulation tool [19]): the authors extended numerical modeling of the semi-infinite cylindrical fibers in 3-D with periodical boundary conditions, and provided original assumptions for the validity of DHM [4] in EMC context. Antennas and propagation (AP) community has also shown a growing interest for the development of 3-D numerical tools aiming at computing the effective properties (i.e. complex permittivity) of composite structure. In this case, heterogeneous substrate made of cubic inclusions has been modeled with finite difference in time domain (FDTD) and software EMPIRE [20], the paper demonstrated the in- 
terest of metallic, cubic, and equally distributed inclusions both from a numerical and an experimental point of views. Finally, an important piece of work was proposed by Baer et al. in [15] since the authors used CST® for large frequency bandwidth extractions of the effective permittivity of solid-liquid composite mixtures (wet snow). This metrological and numerical work involved modeling of periodically aligned inclusions jointly with waveguide and S-

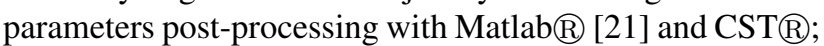
it was restricted and optimized to the frequency band range from 18 to $26 \mathrm{GHz}$. The present work requires time simulations for assessing the SE statistics of 3-D composite materials (e.g. the one illustrated in Fig. 1) for frequency $f \in[0.1 ; 60] \mathrm{GHz}$.

\subsubsection{Methodology}

Following a parallel methodology to works from [15], the main steps of the automated procedure are given as follows:

- Initialization of randomly located spherical inclusions (Matlabß [21]) with given radii, and nontouching or non-intersecting rules.

- Automated geometrical (including FIT meshing) generation of elements (source, probes, matrix basis, conducting inclusions) with CSTR and Matlabß.

- Controlling parametrization and launch of required time simulations (CSTR) and Matlab(R).

- Automated post-treatment of data and computation of $\mathrm{SE}$ of composite materials (Matlabß).

The previous methodology is entirely controlled with Matlabß and allows to propose statistical developments based upon numerical setup depicted in next subsection 2.3.

At that stage, it is important to underline the fact that the generation of the spherical inclusions complies with the previous rules, i.e. randomly given locations of inclusions with non-intersecting spheres (by themselves and/or with boundaries). Without any loss of generality, the last point is defined a priori, but it may be easily modified if needed (e.g. splitting inclusions with boundaries).

\subsection{Numerical setup and SE extraction}

Relying on numerical setups proposed in 2-D for cylindrical semi-infinite inclusions (i.e. fibers) in [4, 12], the numerical test case proposed in this work is illustrated in Fig. 1.

The numerical characteristics of the CSTß simulations are summarized as follows:

- Transient (T-) solver with total duration of simulation $T_{\text {sim }}=0.4 \mathrm{~ns}$; plane wave (PW) excitation from 0.1 to $60 \mathrm{GHz}$.

- Sizes of the composite material are restricted to $1 \times$ $1 \times 6 \mathrm{~mm}^{3}$ parallelepiped (see Fig. 1).

- Meshing: mean value of elementary cells required for solving with T-solver is assessed (see Tab. 1).

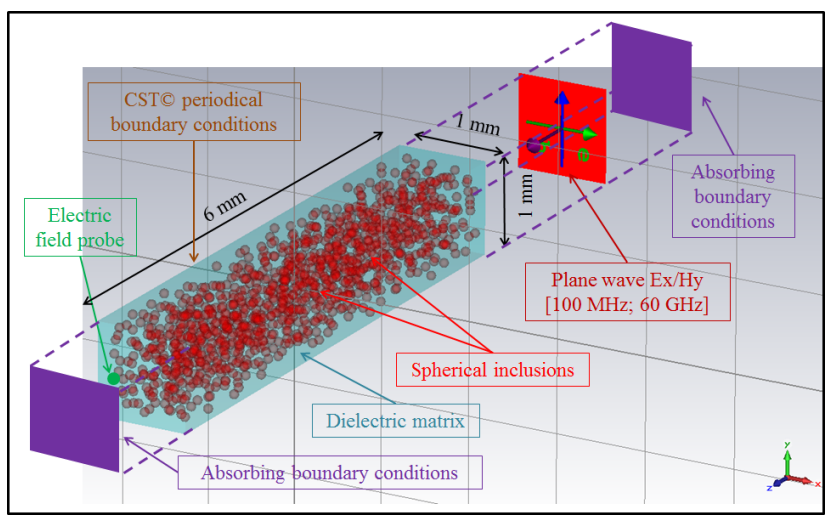

Figure 1: Mockup of numerical setup based upon 3-D CST $\AA$ model of the material (transient FIT solver) including boundary conditions and dimensions. The properties of the two-phases mixture are as follows: $\epsilon_{1}=5 \epsilon_{0}, \sigma_{1}=$ $0 \mathrm{~S} / \mathrm{m}, \epsilon_{2}=\epsilon_{0}, \sigma_{2}=1,000 \mathrm{~S} / \mathrm{m}$, and $\nu=1-\eta=0.10$; where $\epsilon_{0}$ stands for the dielectric permittivity in vacuum.

It is to be noted here that the choice of the elementary composite cell (i.e. $1 \times 1 \times 6 \mathrm{~mm}^{3}$ parallelepiped) was governed by the works proposed in $[4,12,13,17]$ with similar materials and comparable micro-structures.

In order to firstly ensure the required level of geometrical accuracy for meshing purposes (T-solver used in CST $($ ), the following criterion (in \%) is defined for each simulation frequency $f$ at location $z_{p}$ of electric field probe (see Fig. 1)

$$
G_{a}\left(f, z_{p}\right)=100\left|\frac{E\left(f, z_{p}\right)_{a}-E\left(f, z_{p}\right)_{a+10}}{E\left(f, z_{p}\right)_{a+10}}\right|,
$$

where $E\left(f, z_{p}\right)_{a}$ stands for the electric field measured at location $z_{p}$ and frequency $f$ for a meshing quality depending on the averaged ratio between elementary cells used for numerical model and given by $\Delta=\lambda_{0} / a\left(\lambda_{0}\right.$ represents the minimal wavelength of the excitation frequency $f$ ). In this framework, increasing $a$ leads to finer meshing case (here with volumic fraction $\nu=0.01$ and size of spherical inclusions $r=0.050 \mathrm{~mm})$. Thus, $G_{a}\left(f, z_{p}\right)$ provides a quick overview of the relative gap due to a finer description of the geometries. $G_{a}\left(f, z_{p}\right)$ statistics are obtained over the whole frequency band range by extracting the mean and extreme values (i.e. minimum and maximum) of the criterion from relation (4) and for one given location of the probe (see Fig. 1). This is expected that these statistics (i.e. mean, minimum, and maximum) tends to zero over the whole frequency bandwidth when increasing $a$ (see Tab. 1 for results obtained).

As expected, data presented in Tab. 1 clearly justifies the choice of numerical characteristics used for an optimal meshing quality. Indeed, based upon a common geometrical configuration (i.e. one random location of spherical conducting inclusion such as the case described in Fig. 1), 5 different simulations were realized, only crudely increasing the refinement of meshing (from initial $\Delta=\lambda_{0} / 10$ to 


\begin{tabular}{c||c|c|c|c}
\hline Meshing case & $a=10$ & $a=20$ & $a=30$ & $a=40$ \\
\hline Mean gap (\%) & 7.174 & 0.852 & 0.304 & 0.197 \\
\hline Mini. gap (\%) & 0.044 & 0.001 & 0.000 & 0.001 \\
\hline Maxi. gap (\%) & 24.598 & 2.781 & 0.895 & 0.598 \\
\hline
\end{tabular}

Table 1: Statistical (mean, minimum, mini., and maximum, maxi.) relative gaps (see relation (4)) from one meshing test case (see Fig. 1) to another (i.e. from meshing mean elementary cell around $\Delta=\lambda_{0} / a$ to $\Delta=\lambda_{0} /(a+10)$ ).

$\left.\lambda_{0} / 50\right)$. The relative difference between cases $a=40$ and $a=50$ obviously validates the meshing quality (maximum gap $G_{a}\left(f, z_{p}\right)$ below 1\%). In the following, simulations were achieved with $\Delta=\lambda_{0} / 40$ to guarantee a sufficient level of precision for geometries (e.g. micrometric spherical inclusions from 25 to $100 \mu \mathrm{m}$ ); moreover, reference values will be taken from time domain simulations both in order to

- check the validity of theoretical assumptions (MG formalism, see relation (2)),

- calibrate characteristics of effective permittivity for dynamic homogenization process (DHM [4], see subsection 3.3).

In the framework of EMC measurements, shielding effectiveness (SE) is a common parameter allowing to size protective devices. Many experimental and numerical tools exist to properly quantify this output (e.g. reverberation chambers [22]). In this framework, composite materials reinforced with conducting micro-/nano-structures are of great importance, but require a particular care for their characterization [6]. Physically, the phenomena involved in SE may be split into three parts: reflexion on the external surface, absorption and multiple reflexions inside the mixed material. Mathematically speaking, SE characterizes the behavior of a given material subject to electromagnetic (EM) excitation, and it may be defined as the ratio between the magnitude of impinging electric (E-) field $E_{i n c}$ over the transmitted E-field $E_{t}$ at same location $z$ and a given frequency $f$

$$
S E(f, z)=\frac{E_{\text {inc }}(f, z)}{E_{t}(f, z)} .
$$

The proposed numerical tool provides an automated procedure to extract SE for various random (location of inclusions are randomly chosen) configurations and provides SE statistics. In order to enhance the readability of results, and even if SE is classically expressed in $\mathrm{dB}$ (e.g. in EMC community), next section 3 will provide SE data in linear scale following definition in relation (5).

Finally, it is to be noticed SE data in relation (5) are extracted from time domain solver (T-) simulations (FIT) even if the final results are given in frequency domain from Fast Fourier Transformation (FFT, see following Figs. 2-5).

\section{Material characterization at microwave frequency}

\subsection{Validity and relevance of the model}

This part aims to compare results obtained by 3-D modeling procedure given in subsection 2.2 with expectations from classical MG formalism; as aforementioned, this theoretical formulation is defined by a number of assumptions that is sometimes tricky to check (limit for volumic fraction, restriction with regards to frequency...). Due to intrinsic manufacturing constraints, and/or since uncertainties may be inherent during production process (e.g. for the assessment of inclusions' locations, sizes, shapes...), classical mixing rules such as MG and BR may provide limited information, and for instance lack to predict interactions between inclusions. The interested reader may find an example of the variability and random locations of composite materials in [23] where the authors proposed low cost and environmental friendly generation of nanometric inclusions (pseudocubes, rhombohedra, and spinkles particles) for gas sensing aims.

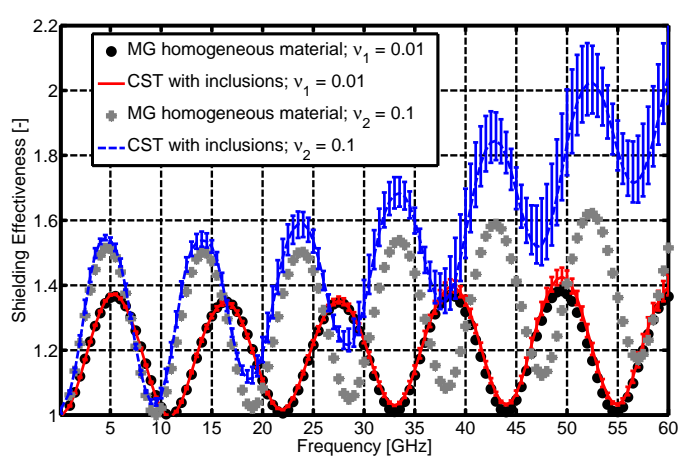

Figure 2: Effect of the volumetric rate of spherical inclusions on shielding effectiveness considering constant radius $r=0.050 \mathrm{~mm}$ and variable volumetric rates: $\nu_{1}=0.01$ and $\nu_{2}=0.10$.

Fig. 2 gives an overview of the SE computed with two different volumetric rates of inclusions (i.e. $\nu_{1}=0.01$ and $\nu_{2}=0.10$ ). In each previous case, the results given by ten simulations from CST $\AA$ are proposed in comparison to the theoretical value given by analytical formulation of field propagation inside an homogeneous medium obtained from MG formalism (see formula (2)). It is to be noted that plain curves (respectively red and blue ones for volumetric rates $\nu_{1}=0.01$ and $\nu_{2}=0.10$ ) are obtained from the ten time domain CST $®$ simulations, and thus from ten different random micro-structures (i.e. ten random sets of inclusions with random locations of spherical inclusions). In this framework, the error bars correspond to minimum and maximum SE extracted from the ten previous simulations at each studied frequency. As it could be expected, the statistical dispersion enhances when increasing the volumetric rates (see results respectively with $\nu_{1}$ and $\nu_{2}$ ). 


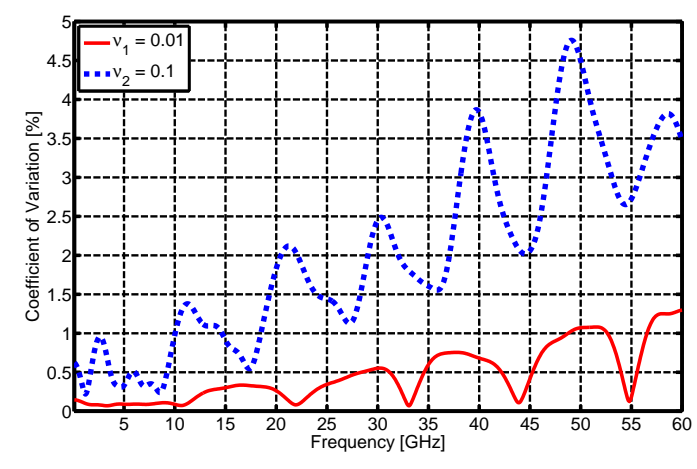

Figure 3: SE coefficient of variation (i.e. rate between SE std and SE mean considering 10 CST $®$ simulations with random location of spherical inclusions; $r=0.050 \mathrm{~mm}$ ) for various volumetric rate $\nu_{1}=0.01$ and $\nu_{2}=0.10$.

\begin{tabular}{c|c|c}
\hline Volumetric rate & CV mean (in \%) & CV std (in \%) \\
\hline \hline$\nu_{1}=0.01$ & 0.467 & 0.350 \\
\hline$\nu_{2}=0.10$ & 2.008 & 1.197 \\
\hline
\end{tabular}

Table 2: Mean and standard deviation (std) of SE coefficient of variation $(\mathrm{CV})$ over the whole frequency bandwidth (i.e. here from 0.1 to $60 \mathrm{GHz}$ ), see Fig. 2.

The coefficient of variation (CV) of SE is given in Fig. 3 relatively to frequency. It is to be noted that $\mathrm{CV}$ is the rate between standard deviation (std) and mean value of SE extracted from 10 time domain 3-D simulations (for each volumic fraction $\nu_{1}$ and $\nu_{2}$ ). Moreover, as expressed in [24], CV may be useful to detect the sensitivity of a given model: high sensitivity is often related to high (greater than $10 \%$ for instance) CV values. Fig. 3 is completed by Tab. 2 which gives respectively mean value and std of CV (in \%) over the whole frequency band range.

Tab. 2 shows both the weak sensitivity of numerical configurations for case $\nu_{1}=0.01$ (low levels of CV) and the relatively higher dispersion in case $\nu_{2}=0.10$, previously observed in Fig. 2. In this context, it is also natural to notice that MG formulation works properly for predicting SE levels in case $\nu_{1}$ whereas the results are similar in case $\nu_{2}$ only up to about $10 \mathrm{GHz}$ (Fig. 2). Obviously, Fig. 3 confirms results obtained in Tab. 2. Moreover, for a given threshold $s$ (e.g. $s=1 \%, 2 \% \ldots)$, CV could provide useful information to detect the range of validity for MG approximation. For instance, assuming $s=0.01$ leads to validate the use of MG formulation almost over the entire bandwidth (from 0.1 to $49 \mathrm{GHz}$ ) for volumetric rate $\nu_{1}=0.01$, whereas 3-D time simulations and MG formula agrees well only up to $10 \mathrm{GHz}$ when increasing volumic fraction to $\nu_{2}=0.10$.

\subsection{Impact of the size of inclusions}

As aforementioned, classical MG mixing rule does not take into account the size of inclusions (see relation (2)). The proposed numerical tool provides trustworthy results to assess the levels of statistical dispersion of results due to variations arounf the size of inclusions.

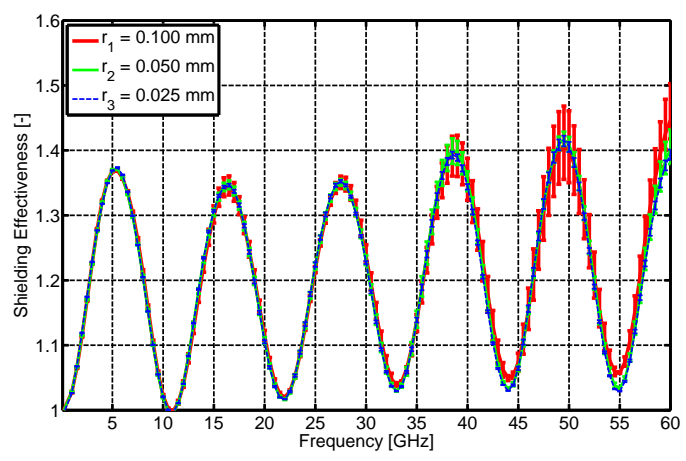

Figure 4: Effect of the sizes of the spherical inclusions on shielding effectiveness considering constant volumetric rate $\nu=0.01$ and variable radii: $r_{1}=0.100 \mathrm{~mm}$, $r_{2}=0.050 \mathrm{~mm}$, and $r_{3}=0.025 \mathrm{~mm}$.

Fig. 4 shows SE statistics taking into account the influence of the radii of spherical inclusions. As expected, increasing the size of inclusions leads to the enhancement of statistical dispersion. Indeed, results for $r_{2}=0.050$ and $r_{3}=0.025 \mathrm{~mm}$ are in accordance with restricted levels of dispersion (i.e. SE extrema are close to mean values obtained from 10 simulations, see error bars and curves in Fig. 4). Contrary to previous cases, spherical inclusions with $r_{1}=0.100 \mathrm{~mm}$ show noticeable dispersion from $15 \mathrm{GHz}$. This may both reveal the domain of validity for MG approximation (depending on the size of the inclusions here) in function of frequency and show the level of interaction between inclusions in function of their corresponding size (i.e. radius here). Obviously, SE extrema is greater in test case $r_{1}$ comparatively to $r_{2} / r_{3}$ configurations; despite all, the averaged SE (curves) follows same trends for weak volumic fraction $\nu=0.01$ (in accordance with comparison between 3-D numerical data and MG formulation in Fig. 2).

\subsection{Optimized homogenization procedure based upon 3-D SE statistics}

This part is devoted to the presentation of results obtained by combining equations (1) and (3). Thus, as expressed in subsection 2.1, DHM should improve the prediction of SE for high frequency band range. The procedure provided in [4] leads to $\alpha=(d / \lambda)^{\gamma}$ (see equation (3 and part 2.1) with $\gamma \approx 2=1.92$ similarly to what was proposed for cylindrical fibers [17]. It is to be noted that $d$ stands for the radius of inclusions (here $d=0.100 \mathrm{~mm}$ is the diameter of spheres).

Fig. 5 demonstrates the validity of the proposed approach and the interest of DHM based upon time domain 3$\mathrm{D}$ simulations. Indeed, the averaged SE allows to extract effective conductivity of the infinite medium (part of effective infinite permittivity $\epsilon_{\infty}$ ). The extrema given by minimum 


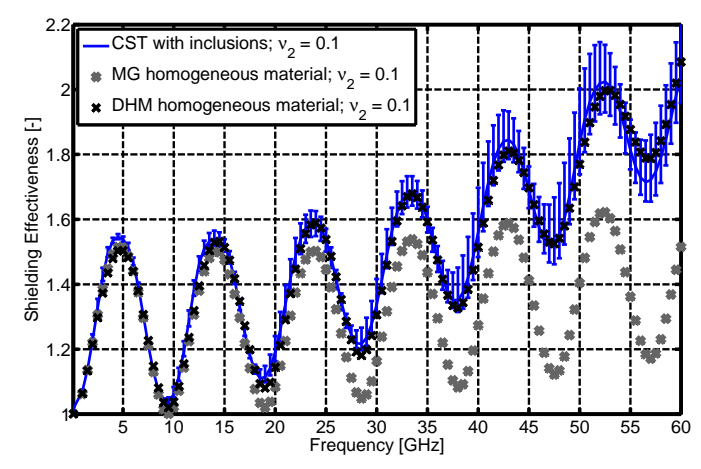

Figure 5: SE statistics from CST® simulations (mean trend and extrema from 10 simulations, blue) in comparison to classical MG (bold gray crosses) formulation and proposed DHM (black crosses) with volumetric rate of inclusions $\nu_{2}=0.10$, and radius $r=0.050 \mathrm{~mm}$.

and maximum SE extracted from CST® simulations offers useful margins (blue in Fig. 5) to validate analytical SE results given by DHM. As depicted in Fig. 5, SE data from DHM procedure (black crosses) are in accordance with expectations from time domain simulations (blue curve and error bars), and improve the prediction from initial MG analytical model (bold gray crosses). Same kind of observation was achieved by varying conductivities and/or volumic fractions of inclusions (data not shown here).

Although the number of simulations is restricted here (10 for each test case) for practical reasons (one time simulation may last between 1 and few hours depending on the size of inclusions and the volumic fraction of inclusions, with 600 data computed between 0.1 and $60 \mathrm{GHz}$ ), bootstrapping procedure [25] may offer interesting prospects to provide trustworthy mean values jointly with high quantiles with a limited number of experiments [26].

\section{Conclusion}

This article is devoted to the extraction of statistical electromagnetic shielding properties of composite biphasic mixtures for EMC purposes. A simulation tool has been developed to assess the variability of random achievement of composite materials. The restrictions due to classical mixing formulas (such as MG for instance) was highlighted, whereas the use of DHM was validated with extrema values provided by time domain simulations over a large frequency bandwidth (here from 0.1 to $60 \mathrm{GHz}$ ). The automation of the entire procedure was described and enabled to feed DHM procedure from mean trend of shielding effectiveness. Indeed, the discrepancy observed between time domain data and MG results were confirmed relying on SE statistics (e.g. coefficient of variation). The entire procedure is controlled with Matlabß and offers a huge flexibility regarding the sizes/shapes of the conducting inclusions, their locations and electrical properties.

Although a priori non-intersecting rules were defined, the proposed methodology may be easily adapted to alternative generation procedures (for instance by defining elementary computational domain with split inclusions at boundary level).

Further works are in progress to enrich this work with alternative inclusions and/or sources jointly with comparisons with measurements. The proposed numerical tool may be useful out of pure EMC applications, for instance regarding antennas and propagation, metrology, or sensing. Without any loss of generality (weak levels of conductivity were assumed for inclusions with $\sigma=1,000 \mathrm{~S} / \mathrm{m}$ ), the proposed methodology might be straightforward applied with automated procedure for "classical" conducting particles, such as carbon mixed materials. Nowadays, complementary works are initiated to consider various species of inclusions with varying conductivity and higher levels (from $10,000$ to $60,000 \mathrm{~S} / \mathrm{m})$.

\section{References}

[1] S. Kozik, M. Binhussain, A. Smirnov, N. Khilo, and V. Agabekov, "Investigation of surface roughness influence on hyperbolic metamaterial performance," Advanced Electromagentics, vol. 3, pp. 6-9, 2014.

[2] P. Tichit, S. Burokur, X. Wu, D. Germain, and A. de Lustrac, "Novel antenna concepts via coordinate transformation," Advanced Electromagentics, vol. 2, pp. 23-32, 2013.

[3] C. Njoku, W. Whittow, and J. Vardaxoglou, "Effective permittivity of heterogeneous substrates with cubes in a 3-d lattice," IEEE Antennas and Wireless Propagation Letters, vol. 10, pp. 1480-1483, 2011.

[4] V. Préault, R. Corcolle, L. Daniel, and L. Pichon, "Effective permittivity of shielding composite materials for microwave frequencies," IEEE Transactions on Electromagnetic Compatibility, vol. 55, pp. 11781186, 2013.

[5] M. Boubekeur, A. Kameni, and L. Pichon, "Modeling of thin heterogeneous sheets in the discontinuous galerkin method for $3 \mathrm{~d}$ transient scattering problems," European Physical Journal Applied Physics, vol. 73, pp. 1-5, 2016.

[6] D. Micheli, M. Marchetti, R. Pastore, A. Vricella, G. Gradoni, F. Moglie, and V. Mariani Primiani, "Shielding effectiveness of carbon nanotube reinforced concrete composites by reverberation chamber measurements," in Electromagnetics in Advanced Applications (ICEAA), 2015 International Conference on, pp. 145-148, 2015.

[7] C. Baer, T. Jaeschke, P. Mertmann, N. Pohl, and T. Musch, "A mmwave measuring procedure for mass flow monitoring of pneumatic conveyed bulk materials," IEEE Sensors Journal, vol. 14, pp. 3201-3209, 2014. 
[8] A. Arslan, H. Wang, J. Pulliainen, and M. Hallikainen, "Effective permittivity of wet snow by using two-phase strong fluctuation theory with nonsymmetrical inclusions," in International Symposium on Geoscience and Remote Sensing 2000, pp. 15191521, 2000.

[9] P. Uhd Jepsen, B. Fisher, A. Thoman, H. Helm, J. Suh, R. Lopez, and R. Haglund, "Metal-insulator phase transition in a vo2 thin film observed with terahertz spectroscopy," Physical Review B, vol. 74, pp. 1-9, 2006.

[10] M. Koledintseva, R. DuBroff, and R. Schwartz, "A maxwell garnett model for dielectric mixtures containing conducting particles at optical frequencies," Progress In Electromagnetics Research (PIER), vol. 63, pp. 223-242, 2006.

[11] M. Koledintseva, R. DuBroff, and R. Schwartz, "Maxwell garnett rule for dielectric mixtures with statistically distributed orientations of inclusions," Progress In Electromagnetics Research (PIER), vol. 99, pp. 131-148, 2009.

[12] V. Préault, R. Corcolle, L. Daniel, and L. Pichon, "Shielding effectiveness of composite materials: effect of inclusion shape," IEEE Transactions on Magnetics, vol. 49, pp. 1941-1944, 2013.

[13] V. Préault, Méthode d'homogénéisation pour la modélisation électromagnétique de matériaux composites. PhD Thesis (in French), Université Paris Sud, 2013.

[14] "CST - computer simulation technology." https: / / WWW. cst. com/. Accessed: Oct. 2016.

[15] C. Baer, B. Hattenhorst, C. Shultz, B. Will, I. Rolfes, and T. Musch, "Analysis of composite materials with periodically aligned inclusions using $3 \mathrm{~d}$ field simulations," in GeMiC 2016, pp. 120-123, 2016.

[16] T. Mori and R. Tanaka, "Average stress in matrix and average elastic energy of materials with misfitting inclusions," Acta Metall. Mater, vol. 21, pp. 597-629, 1973.

[17] Y. Liao, G. Shi, L. Yuan, and X. Ying, "Equivalent modeling of the microwave dielectric properties for fiber reinforced shielding composites," in 7th Asia Pacific International Symposium on Electromagnetic Compatibility, 2016.

[18] "COMSOL multiphysics." https://www . comsol.fr/. Accessed: Oct. 2016.

[19] "FEKO em simulation solver." https://www . feko.info/. Accessed: Oct. 2016.

[20] "EMPIRE xpu." http: / / www . empire. de/. Accessed: Oct. 2016.
[21] "MATLAB - mathworks." https://fr. mathworks.com/products/matlab/. Accessed: Oct. 2016.

[22] A. Gifuni, A. Sorrentino, A. Fanti, G. Ferrara, M. Migliaccio, G. Mazzarella, and F. Corona, "On the evaluation of the shielding effectiveness of an electrically large enclosure," Advanced Electromagentics, vol. 1, pp. 84-91, 2012.

[23] G. Bailly, J. Rossignol, B. de Fonseca, P. Pribetich, and D. Stuerga, "Microwave gas sensing with hematite: Shape effect on ammonia detection using pseudocubic, rhombohedral, and spindlelike particles," ACS Sensors, vol. early access, 2016.

[24] S. Lalléchère, B. Jannet, P. Bonnet, and F. Paladian, "Sensitivity analysis to compute advanced stochastic problems in uncertain and complex electromagnetic environments," Advanced Electromagentics, vol. 1, pp. 13-23, 2012.

[25] S. Lalléchère, C. Kasmi, J. Lopes Esteves, S. Girard, P. Bonnet, and F. Paladian, "Optimization of emc shielding procedures by means of statistical resampling: from mean trends to reliability assessments," in 4th Advanced Electromagnetics Symposium, AES 2016, 2016.

[26] C. Kasmi, S. Lalléchère, J. Lopes Esteves, S. Girard, P. Bonnet, F. Paladian, and E. Prouff, "Stochastic emc/emi experiments optimization using re-sampling techniques," IEEE Transactions on Electromagnetic Compatibility, vol. 58, 2016. 